\title{
Effect of recombinant human bone morphogenetic protein 2 (rhBMP-2) on equine bone formation: A preliminary in vitro and in vivo evaluation
}

\author{
Michelle A. Jackson 1,2,3, Anton E. Fürst' ${ }^{1,2}$, Sabine Koch ${ }^{4}$, Brigitte von Rechenberg ${ }^{1,4}$, Peter J. Richards ${ }^{1,5}$ \\ 1 Center for Applied Biotechnology and Molecular Medicine (CABMM), University of Zurich, Zurich, Switzerland \\ 2 Equine Department, Vetsuisse-Faculty, University of Zurich, Zurich, Switzerland \\ 3 Graduate School for Cellular and Biomedical Sciences, University of Bern, Bern, Switzerland \\ 4 Musculoskeletal Research Unit (MSRU), University of Zurich, Zurich, Switzerland \\ ${ }^{5}$ Bone and Stem Cell Research Group, University of Zurich, Zurich, Switzerland
}

\begin{abstract}
Summary: Bone morphogenetic protein 2 (BMP-2) is a potent inducer of osteoblast-mediated bone formation and is used in orthopaedic applications in humans. This study was performed to evaluate (1) recombinant human BMP-2 (rhBMP-2) in clinical cases of subchondral cystic lesion (SCL) in horses and (2) the osteoinductive effects of rhBMP-2 treatment in vitro on primary equine osteoblasts. Three horses with SCLs of the proximal interphalangeal (PIP) joint and associated lameness were treated by transcortical debridement and implantation of a collagen sponge containing rhBMP-2. In the in vitro studies, equine osteoblasts were cultured with rhBMP-2 to assess the effects of rhBMP-2 on their differentiation and mineralization. The treatment of SCLs with rhBMP-2 was well tolerated by all the horses, and improvements in subchondral bone radiographic appearance and lameness score were evident between 7 and 10 months post-surgery. Equine osteoblasts treated with rhBMP-2 demonstrated enhanced mineralization as evidenced by increases in Alizarin Red S staining. This was accompanied by significant increases in alkaline phosphatase (ALP) expression and activity, and a significant decrease in secreted osteocalcin levels. In conclusion rhBMP-2 can be used to assist in subchondral bone repair in horses, and we provide in vitro evidence to suggest that its influence on bone formation is mediated through enhanced osteoblast activity.
\end{abstract}

Keywords: bone morphogenetic protein 2 (BMP-2), osteoinductivity, bone formation, equine osteoblasts, subchondral cystic lesions (SCLs)

Citation: Jackson M. A., Fürst A. E., Koch S., von Rechenberg B., Richards P. J. (2017) Effect of recombinant human bone morphogenetic protein 2 (rhBMP-2) on equine bone formation: A preliminary in vitro and in vivo evaluation. Pferdeheilkunde 33, 424-432; DOI $10.21836 /$ PEM20170501

Correspondence: Michelle Jackson. Dr. med. vet., Dipl. ECVS, Vetsuisse Faculty Zurich, Equine Department, Winterthurerstrasse 260, 8057 Zurich, Switzerland; E-mail: mjackson@vetclinics.uzh.ch

\section{Introduction}

Subchondral cystic lesions (SCLs) occur in horses as soft tissue-filled defects in subchondral bone. Although their origin is unclear, SCLs often pose a substantial problem because they stimulate inflammation, are covered with degenerated cartilage, and often result in lameness (von Rechenberg et al. 2000). SCLs are found in many locations, most commonly the medial femoral condyle, followed by the phalanges, carpal bones, 3rd metacarpal and metatarsal bones, tibia, radius, talus, sesamoid bones, and humerus (von Rechenberg et al. 1998). Surgical debridement is the standard surgical procedure used to treat equine SCLs and involves an arthroscopic or transcortical approach for curettage of the cyst content and cyst lining (Fürst et al. 1997, Story and Bramlage 2004). This may be accompanied by filling the defect with agents designed to enhance bone formation, including osteoinductive proteins. Parathyroid hormone peptide $\left(\mathrm{PTH}_{1-34}\right)$ in fibrin hydrogel administered locally into SCLs led to a $78.6 \%$ recovery of the treated horses (Jackson et al. 2012). Although the exact mechanism of the anabolic effect with PTH is not clear, its main function is attributed to stimulating osteoblast proliferation (Rubin and Bilezikian 2003). Treatment of equine SCLs using insulin-like growth factor (IGF)-1 in combination with allogeneic chondrocytes led to improved healing in the short-term (8 weeks) and long-term (8 months) follow-up with a successful outcome in $80 \%$ of horses over 3 -years-of- age, even in the presence of joint degeneration (Ortved et al. 2012). It has been suggested that horses older than three years tend to be more refractory to the previously described treatments; Smith et al. (2005) found that only $35 \%$ of horses older than 3 years became sound after surgical debridement of an $\mathrm{SCL}$ in the medial femoral condyle.

More recently, several studies examined the potential beneficial effects of bone morphogenetic protein (BMP)-2 in the repair of equine osteochondral defects (Seo et al. 2013, Seo et al. 2015, Tsuzuki et al. 2013). BMP-2 is a member of the BMP family, and is predominantly synthesized by osteoblasts (Smith et al. 2008). Due to its osteoinductive effects (Devescovi et al. 2008), it is approved as an alternative for cancellous bone grafts in humans in special indications (Govender et al. 2002, Mont et al. 2004). Previous studies demonstrated that recombinant human BMP-2 (rhBMP-2) loaded scaffolds accelerated bone regeneration in equine bone defect models (Perrier et al. 2008, Seo et al. 2014). Gelatin $\beta$-tricalcium phosphate ( $\beta$-TCP) sponges loaded with rhBMP-2 also enhanced healing of osteochondral defects in horses (Seo et al. 2013, Seo et al. 2015, Tsuzuki et al. 2013). However, as rhBMP-2 was administered in combination with multipotent stromal cells (MSCs) and platelet rich plasma (PRP), its actual contribution to osteochondral repair in these models remains unclear. Moreover, although the effect of human BMP-2 on 
the osteogenic differentiation of equine bone marrow-derived MSCs (BMSCs) was described (Carpenter et al. 2010, Glynn et al. 2013), its effects on equine osteoblasts has yet to be determined.

The aim of the present study was to evaluate the clinical outcome of three equine cases with SCLs following combined treatment with debridement and collagen sponges containing rhBMP-2. Furthermore, in vitro studies were undertaken to assess the effects of rhBMP-2 on the differentiation of equine osteoblasts and on induction of mineralization.

\section{Material and methods}

Recombinant human BMP-2 (rhBMP-2) was purchased as part of the InductOs $\mathrm{kit}^{\mathrm{a}}$ and used in accordance with the manufacturer's instruction. For in vitro studies, reconstituted rhBMP2 was added directly to culture medium at final concentrations of $10,50,100$ or $200 \mathrm{ng} / \mathrm{ml}$. For treatment of equine SCLs, rhBMP-2 $(0.5 \mathrm{mg} / \mathrm{ml})$ was evenly applied to a $1 \mathrm{~cm}^{2}$ bovine Type I absorbable collagen sponge (ACS) for at least 15 minutes. Up to two sponges were used per defect depending on the size of the SCL.

\section{Surgical treatment of equine SCLs}

Horses included in the study were referred to the Clinic for Equine Surgery, University of Zurich, between January 2014 and December 2015 because of a forelimb lameness attributable to an SCL in the proximal interphalangeal (PIP) joint (either located distally in the proximal phalanx $(\mathrm{P} 1)$ or proximally in the middle phalanx (P2)). Diagnosis was performed by diagnostic anaesthesia and digital radiography. Cases of SCLs and concomitant fissures, treated with additional cortex screws, were excluded from the study. In all cases, computed tomography (CT) was performed under general anaesthesia in lateral recumbency using a 40-slice helical CT scanner ${ }^{c}$ to confirm the diagnosis and guide the surgical debridement. After native CT, the surgical area was aseptically prepared and surgically draped. A 3.2-mm hole was first drilled transcortically into the cyst, in a predetermined direction and depth. Then the hole enlarged to 4.5 or $5.5 \mathrm{~mm}$. Arthroscopic, thin curettes were used to remove all content and lining of the cyst, and the cavity flushed with lactated Ringer's solution. Pre-soaked collagen sponges containing rhBMP-2 (InductOs) ${ }^{a}$ were then inserted into the cavity and the skin routinely closed. Phenylbutazone $(2.2 \mathrm{mg} / \mathrm{kg} \mathrm{sid})^{\mathrm{d}}$ was administered orally for the first 4-6 days, and horses were confined to box rest for 30 days. Thereafter, horses were turned out into paddocks $\left(6 \mathrm{~m}^{2}\right)$ for up to 4 months before being allowed to resume normal activities. Complete lameness examination and radiography of the surgical site were performed between 7 and 10 months after surgery.

\section{Isolation and culture of equine osteoblasts}

Cancellous bone was collected aseptically from the 3rd metacarpal bones of 5 healthy, Warmblood horses (5-12-years of age) slaughtered for reasons unrelated to this study. Equine osteoblasts were harvested from bone explants using a com- bination of enzymatic digestion and outgrowth as previously described (McCarthy et al. 1988). Bone explants were then transferred to sterile culture dishes and incubated in normal growth medium consisting of Dulbecco's modified eagle medium (DMEM) e supplemented with 10\% foetal bovine serum (FBS)e, $1 \%$ penicillin/streptomycine, $0.3 \mathrm{mg} / \mathrm{ml}$ Fungizonee and $50 \mathrm{mg} / \mathrm{ml}$ ascorbic acidf at $37^{\circ} \mathrm{C} / 5 \% \mathrm{CO}_{2}$. Osteoblast differentiation was induced using osteogenic medium $(\mathrm{OM})$ consisting of alpha-minimum essential medium $(M E M)^{\dagger}$, $10 \% \mathrm{FBS}^{2}$, $1 \%$ penicillin/streptomycine, $50 \mu \mathrm{M}$ ascorbic acid ${ }^{f}$, $10 \mathrm{mM} \beta$-glycerophosphate ${ }^{f}$ and $100 \mathrm{nM}$ dexamethasonef. Cells were also incubated in osteogenic medium devoid of either dexamethasone (OM-D), or in non-osteogenetic medium, devoid of both dexamethasone and $\beta$-glycerophosphate (OM-D/BG). The latter were the control cells. Where stated, rhBMP-2 was included at the indicated concentrations.

\section{Assessment of osteoblast differentiation and mineralization}

Mineralization was assessed at 10 and 12 days post-osteogenic induction using Alizarin Red $\mathrm{S}^{f}$ staining as previously described (Cheng et al. 2003). ALP activity and secreted osteocalcin were measured in cell culture supernatants at selected time points using enzyme-linked immunosorbent assays (ELISAs) specific for bone-specific ALP (BAP EIA Kit)g and intact osteocalcin (Osteocalcin EIA Kit) ${ }^{9}$. Gene expression levels of COL1A2, RUNX2 and ALP were measured in cells at 2 and 5 days post-osteogenic induction using reverse transcription quantitative polymerase chain reaction (RT-qPCR) as previously described (Theiss et al. 2015). Gene expression levels of COL1A2 and RUNX2 were determined using TaqMan Gene Expression Assayse. Ec03469522_ml (COL1A2) and Ec03469742_ml (RUNX2) and values normalized to GAPDH (Ec03210916 gH). ALP expression was determined by SYBR Greene RT-qPCR (Sun et al. 2015), using the following primer pair; forward 5'-GACATGACCTCCCAGGAAGA-3' and reverse 5'-GCAGTCAAGGGCTTCTTGTC-3'. Values were normalized to GAPDH (forward: 5'-ATCACTGCCACCCAGAAGAC-3'; reverse: 5'-GTCAGCTTCCCATTCAGCCTC-3'). All data were expressed as fold change as compared to cells incubated in non-osteogenic medium (control) at day 2 or day 5 using the comparative CT method.

\section{Statistical Analysis}

All statistical analyses were performed using SPSS 23. $0^{\mathrm{h}}$. Parametric analysis of normally distributed data was performed using one-way analysis of variance (ANOVA) followed by Tukey's post-hoc test. In all cases, a p-value of $<0.05$ was considered statistically significant, and all data were expressed as mean \pm standard deviation (S.D.).

\section{Results}

Treatment of SCLs with rhBMP-2

Three horses with a total of 5 SCLs (2 horses affected bilaterally) met the inclusion criteria. The first horse was a 2.5 -yearold mare with intermittent lameness (grade 2/5, AAEP) (Keegan et al. 2010) of the right forelimb, which was abolished 
after a low palmar digital nerve block. On x-ray, an SCL was visible in the distal proximal phalanx $(\mathrm{P} 1)$ of the right forelimb (Figure 1A). CT imaging confirmed a SCL with peripheral sclerosis and periosteal reactions in the medial condyle of the distal P1 close to the PIP joint (Figure 1B). The cyst was then debrided and filled with InductOs (1 mg rhBMP-2), and radiographic analysis performed 2 days later (Figure 1C). At 9 months after surgery, the horse showed no signs of lameness, and radiographic examination confirmed the defect was less radiolucent and less delineated, suggesting progressive bone formation (Figure 1D). At 18 months after surgery, the horse still remained free of lameness and was regularly being used as pleasure horse.
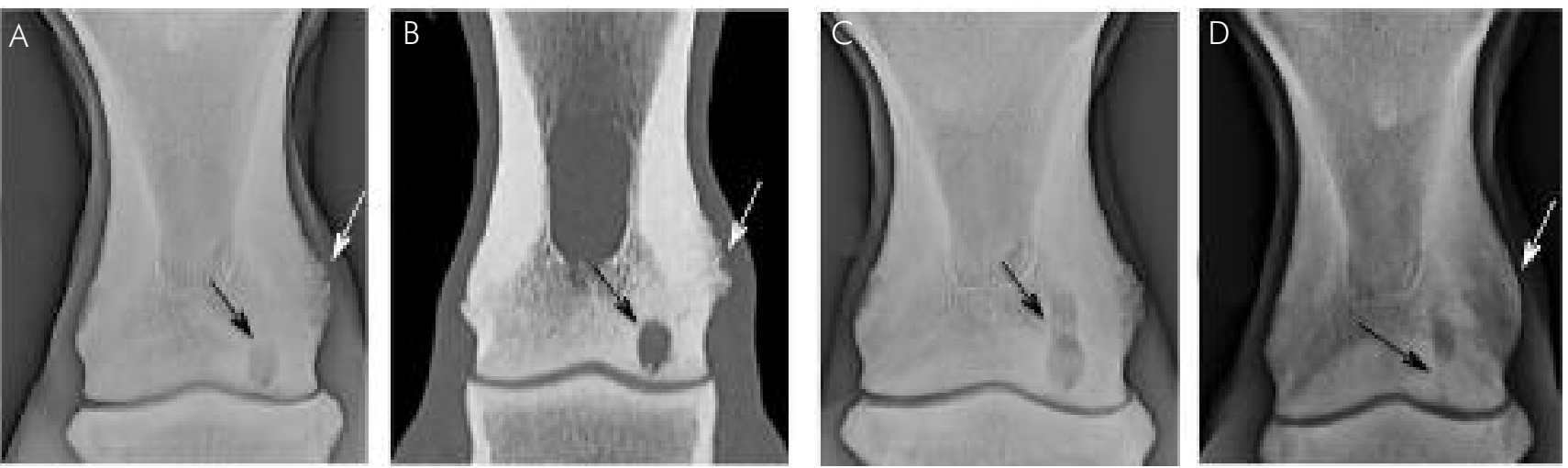

Fig. 1 (A) Dorsopalmar radiographic view of the right PIP joint of Case 1. A SCL is visible in the medial condyle of the distal P1 as an oval and smooth defined radiolucent area (black arrow). Irregular periosteal reactions are visible on the medial distal P1 in the area of the origin of the medial collateral ligament of the PIP joint (white arrow). On dorsal plane CT image (B), a round SCL with peripheral sclerosis (black arrow) as well as the periosteal reactions (white arrow) are visible. (C) Dorsopalmar radiographic view of the right PIP joint 2 days after surgery. The drill entry is indicated with an arrow. (D) Radiographic view at 9 months after surgery. A progressive filling of the lesion is evident close to the joint (black arrow) and the periosteal reactions are smooth (white arrows).
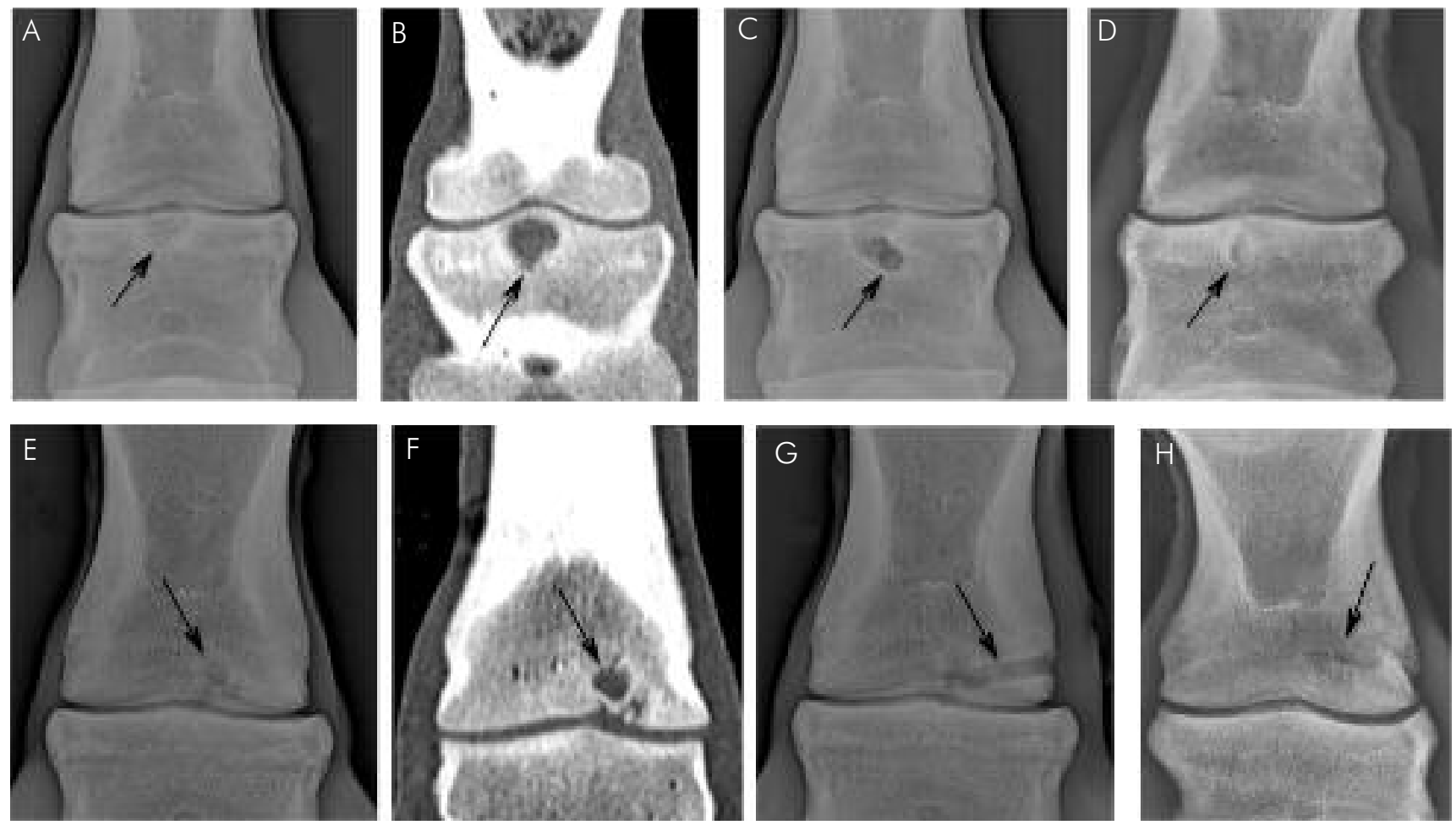

Fig. 2 Dorsopalmar radiographic views of the PIP joint of both forelimbs of Case 2. On the left side, a large SCL is visible sagittal in the proximal P2, surrounded by a sclerotic $\operatorname{rim}(\mathrm{A})$; On the right side, multiple smaller radiolucent areas are visible parasagittal medial, in the distal $\mathrm{P1}$, in close proximity to the PIP joint (E). Dorsal plane CT images confirmed a large SCL on the left side (arrow) (B) and a smaller, irregular one on the right side (arrow) (F). The drill canal (arrows) is visible on the dorsopalmar radiographic views of the left (C) and right (G) PIP joint 2 days after debridement. (D, H) Same radiographic views 10 months after surgery, showing progressive filling of both defects (arrows). 

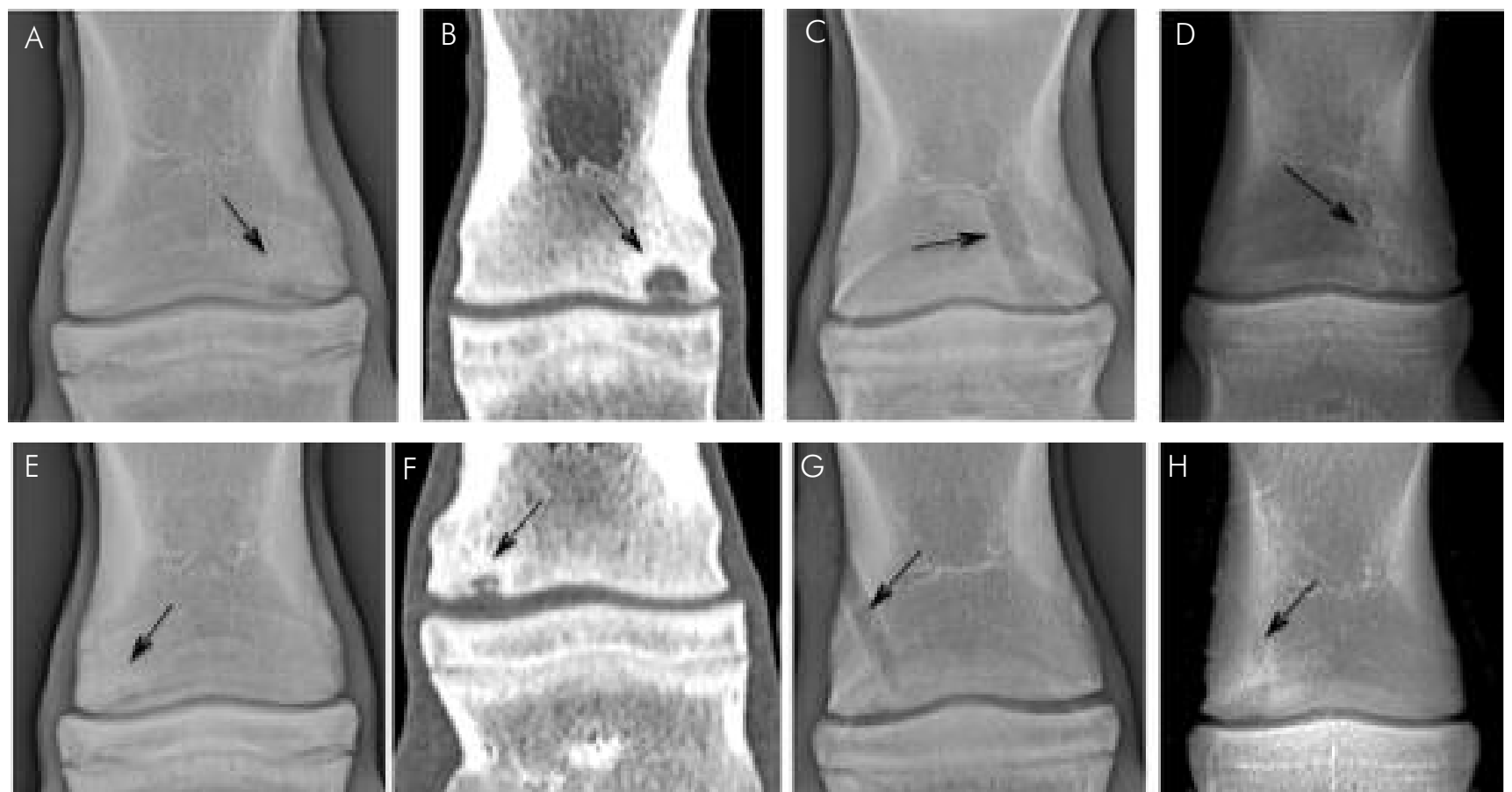

Fig. 3 Dorsopalmar radiographic views of the PIP joint of both forelimbs of Case 3. On the right side (A), an ill-defined radiolucent region in the distal lateral condyle of $\mathrm{Pl}$ is visible (black arrow). On the left (E), a similar but smaller radiolucency is visible on the distal medial $\mathrm{Pl}$ (black arrow). The dorsal plane CT images revealed a round SCL (arrow) on the right P1 (B), and a smaller, irregular SCL (arrow) on the left Pl (F). Both defects were debrided as shown in the dorsal radiographic views of the PIP joints 2 days after surgery, where the drill canal is visible on the right $(C)$ as well as on the left $(G)$ side. At 7 months after surgery, the same radiographic views revealed progressive filling of the drill canal and SCLs in both forelimbs (D,H, arrows).

nation showed no lameness, and radiographic examination showed a progressive filling of the defects in both the left (Figure 2D) and right (Figure 2H) limbs. At 15 months after surgery, the horse still remained free of lameness and was regularly being used for dressage.

The third horse was a 5-month-old filly with moderate, acute lameness (grade 3/5) of the right forelimb, which was abolished after a palmar abaxial sesamoid block. On radiography, an ill-defined radiolucent region was visible in the distal P1 of the right forelimb (Figure 3A), and a smaller one on the distal medial P1 of the left side (Figure 3E). CT examination confirmed a round $\mathrm{SCL}$ on the right $\mathrm{Pl}$ (Figure 3B) and a smaller, irregular one on the left P1 (Figure 3F). Both SCLs were debrided and filled with InductOs (1 mg rhBMP-2 each) and control examinations performed two days after surgery (Figures $3 \mathrm{C}$ and $\mathrm{G}$ ). Radiographic examination 7 months after surgery showed a progressive filling of the both defects (Figures 3D and $\mathrm{H}$ ), and the filly was still free of lameness at 13 months post-surgery.

\section{Effect of rhBMP-2 on equine osteoblast differentiation}

Primary equine osteoblasts were incubated in osteogenic medium in the presence or absence of rhBMP-2 for up to 12 days and their ability to mineralize determined by Alizarin Red $S$ staining. Marked increases in mineral production in response to increasing concentrations of rhBMP-2 were observed already after 10 days of osteoblast differentiation in osteogenic medium either with dexamethasone (OM) or without dexamethasone (OM-D) (Figure 4A). By day 12, positive staining for Alizarin Red $\mathrm{S}$ was evident in all cultures treated with complete osteogenic medium (OM) (Figure 4B). By contrast, mineral deposition in cultures incubated without dexamethasone $(O M-D)$ was only evident following treatment with the

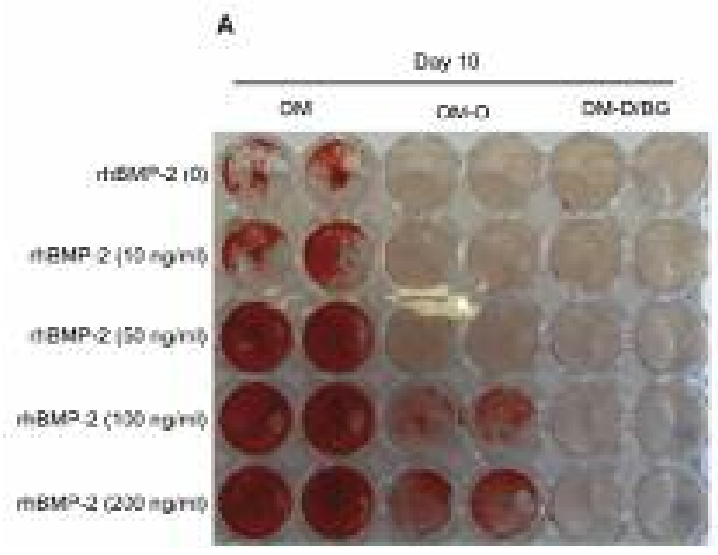

B

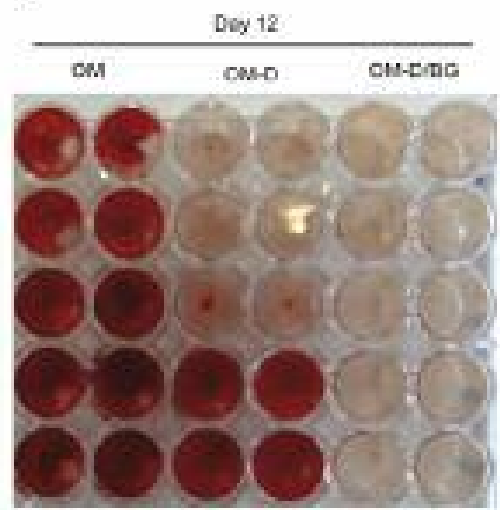

Fig. 4 Analysis of mineralization. Equine osteoblasts were incubated with recombinant BMP-2 in the presence of osteogenic medium (OM) for 10 (A) and 12 (B) days and mineral deposition visualized by Alizarin red staining. $O M$, complete osteogenic medium; OM-D, without dexamethasone; OM-D/BG, without dexamethasone and $\beta$-glycerophosphate. 
two highest concentrations of rhBMP-2 (Figure 4B). In all cases, removal of $\beta$-glycerophosphate from the osteogenic medium (OM-D/BG) prevented mineral formation, even in the presence of rhBMP-2.

Treatment of equine osteoblasts with rhBMP-2 also led to significant increases in ALP activity levels (Figure 5A). The greatest effects were observed using 50 and $100 \mathrm{ng} / \mathrm{ml} \mathrm{rhBMP}-2$, with ALP activity showing the greatest increase at day 5 . We also investigated the effect of rhBMP-2 on the level of secreted osteocalcin, an important mediator of mineral formation. Comparable levels of osteocalcin were observed between all treatment groups at day 4 after the addition of osteogenic medium (Figure 5B). However, by day 7, osteocalcin levels had significantly decreased in cultures treated with 50 or 100 $\mathrm{ng} / \mathrm{ml} \mathrm{rhBMP}-2$, and remained significantly reduced throughout the course of the study.

We used RT-qPCR in order to assess the influence of rhBMP-2 on various genetic markers associated with osteogenesis inclu- ding Runt-related transcription factor 2 (RUNX-2), alkaline phosphatase (ALP) and collagen type 1 alpha 2 chain (COL1A2). Expression levels of RUNX2 at days 2 and 5 were significantly increased in all treatment groups as compared to control cells cultured in non-osteogenic medium (Figure 6A). However, treatment with rhBMP-2 failed to significantly elevate these levels further. By contrast, significant increases in ALP expression levels in cells treated with $100 \mathrm{ng} / \mathrm{ml}$ rhBMP-2 were already evident by day 2 (Figure 6B). Although ALP expression levels had significantly increased in all groups as compared to controls by day 5 , cells treated with $100 \mathrm{ng} / \mathrm{ml}$ rhBMP- 2 continued to express significantly greater levels of ALP than all other groups. Expression levels of COL1A2 at day 2 were comparable between treatment groups, although significantly reduced as compared to controls (Figure 6C). By day 5, although expression levels of COL1A2 had increased by 4 -fold in control cells, expression levels remained significantly reduced in all treatment groups. Furthermore, cells treated with $100 \mathrm{ng} / \mathrm{ml}$ rhBMP2 expressed significantly less COL1A2 as compared to untreated cells, or cells treated with $10 \mathrm{ng} / \mathrm{ml}$ rhBMP-2.
Fig. 5 Analysis of ALP activity and osteocalcin expression. Equine osteoblasts were incubated with recombinant BMP-2 in complete osteogenic medium (OM) for up to 10 days and ALP activity (A) and osteo-calcin secretion (B) measured at selected time points. Data is expressed as mean \pm S.D., and statistical significance determined by one-way ANOVA. * $\mathrm{P}<0.01$ as compared to cells treated with $\mathrm{OM}$ only.
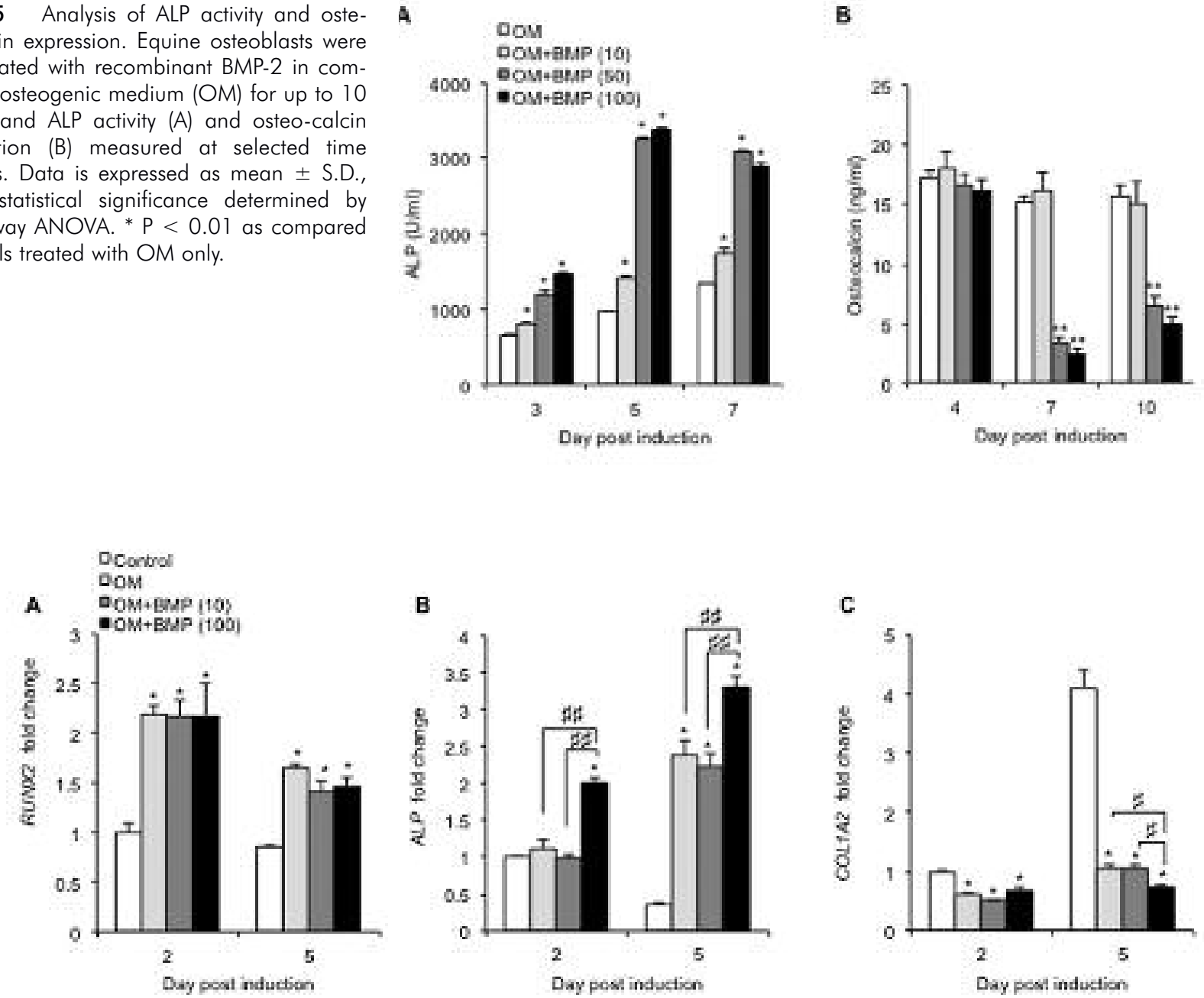

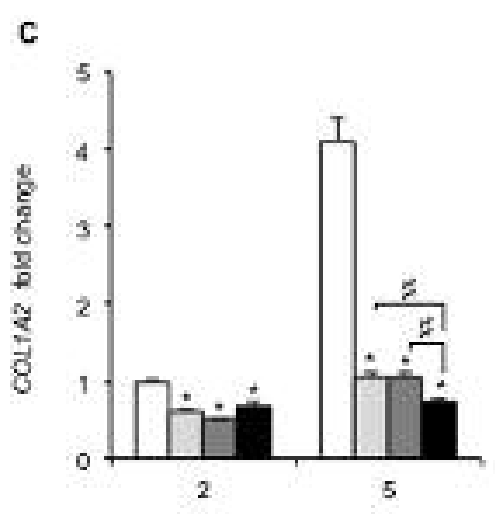

Day post induction

Fig. 6 RT-qPCR analysis of osteogenic markers. Equine osteoblasts were incubated with recombinant BMP-2 (number in parentheses refers to the concentration used) in complete osteogenic medium (OM) for up to 5 days and RNA isolated and the expression levels of RUNX2 (A), ALP (B), and COL1A2 (C) determined by RT-qPCR at selected time points. Data was normalized to GAPDH and expressed as fold change as compared to cells treated with OM only (value 1) using the comparative CT method. Data is expressed as mean \pm S.D., and statistical significance determined by one-way ANOVA. ${ }^{*} \mathrm{P}<0.01$ as compared to cells in non-osteogenic medium (control). \# $\mathrm{P}<0.05$, \#\# $\mathrm{P}<0.01$. 


\section{Discussion}

Subchondral cystic lesions (SCLs) of the proximal interphalangeal (PIP) joint occur in the medial or lateral condyle of the proximal phalanx, or close to the proximal articular surface of the middle phalanx (Redding 2007). Various pathogenic processes have been implicated in the occurrence of SCLs, with trauma being the likely cause in mature horses (Sherlock and Mair 2011) and osteochondrosis in juvenile horses (Verschooten and De Moor 1982). In the cases described herein, a developmental aetiology was suspected based on the young age and the bilateral occurrence in two of the three horses affected, and lack of any history of trauma (Verschooten and De Moor 1982).

Overall, the prognosis for return to performance for horses with phalangeal SCLs ranges from 30-90\%, but is largely dependent on the age, treatment regime, location of the $\mathrm{SCL}$, and concurrent osteoarthritis (Sherlock and Mair 2011 ). Smith et al. (2005) have suggested that older horses, and those with pre-existing osteoarthritis, have a significantly poorer prognosis following surgical debridement of femoral SCLs, with only $35 \%$ of horses older than 3 years returning to soundness after debridement. Similarly, phalangeal SCLs have a poorer prognosis in older horses than in younger horses (Howard et al. 1995). Conservative or surgical interventions are used to treat SCLs in the PIP joint. Conservative treatment consists of a rest period out at grass, followed by gradual return to full training (Jeffcott and Kold 1982), or controlled exercise and systemic administration of NSAIDs, often in combination with intra-articular medication (Trotter and Dyson 2003). However, since the outcome of conservative treatment of SCLs is generally poor, surgical intervention is currently the recommended treatment option (Mcllwraith 2013), especially in older horses, or in horses that are refractory to conservative treatment (Ortved et al. 2012, Smith et al. 2005). Since SCLs of the PIP joint are not arthroscopically accessible, surgical debridement is usually performed through a transcortical approach (Fürst et al. 1997, Mettenleiter 2014). In order to expedite new bone formation within the defect sites, the use of various osteoinductive biomaterials including calcium phosphate (Fürst et al. 1997, Mettenleiter 2014) and $\mathrm{PTH}_{1-34}$-enriched fibrin hydrogel (Fürst et al. 1997) have previously been investigated. Fürst et al. (1997) demonstrated full recovery of SCLs in the distal aspect of the proximal phalanx in 2 out of 3 foals treated with transcortical debridement and filling of the defect with tricalcium phosphate granules. The same group also demonstrated complete healing of an SCL of the distal aspect of the proximal phalanx in a filly following debridement and filling of the defect with $\mathrm{PTH}_{1-34}$ enriched fibrin hydrogel (Fürst et al. 2007).

Recent studies have highlighted the beneficial effects of using rhBPM-2 to repair various experimentally induced equine bone and articular cartilage defects. Tsuzuki et al. (2012) demonstrated that rhBMP-2/ $\beta$-TCP sponges could significantly enhance bone regeneration as compared to either component alone. Moreover, significant improvements in the healing of surgically induced bone and osteochondral defects were demonstrated following treatment with rhBMP-2/ $\beta$-TCP sponges in combination with MSCs (Seo et al. 2014), or with MSCs and platelet rich plasma (Seo et al. 2013, Seo et al.
2015, Tsuzuki et al. 2013). In the present study, we set out to evaluate rhBMP-2 as an adjunct to debridement in the treatment of clinical cases of SCLs in three horses. We hypothesized that, when applied locally to the SCL, rhBMP-2 would facilitate filling of the entire cavity with new bone tissue via its ability to stimulate osteoblast-mediated mineralization. In order to accomplish this, debrided subchondral defects were filled with a collagen sponge containing up to $2 \mathrm{mg}$ of rhBMP2 . In all cases, treatment resulted in increased bone density, decreased cyst size, and an absence of lameness. Therefore, these findings support the use of rhBMP-2 as a possible new treatment strategy for cystic lesions in the pastern joint of horses. However, further studies using larger patient cohorts and appropriate controls (e.g. carrier alone) are clearly needed in order to fully assess the clinical efficacy of rhBMP-2 in treating cystic lesions in horses. Ideally, comparisons should also be made between horses treated with rhBMP-2 and those treated conservatively over extended periods of time.

The osteoinductive effects of rhBMP-2 are primarily mediated through direct stimulation of osteoblasts and their precursors (Kirker-Head 1995, Sandhu et al. 1997). Treatment of equine subchondral cystic lesions with rhBMP-2 would therefore be expected to influence the differentiation and mineralization of resident BMSC-derived osteoblasts. However, although the role of rhBMP-2 in equine BMSC osteogenesis has previously been described (Carpenter et al. 2010, Glynn et al. 2013, Murray et al. 2010), its influence on equine osteoblast differentiation has up until now remained undetermined. Findings from our in vitro studies confirmed that rhBMP-2 stimulated equine osteoblast mineralization as evidenced by marked increases in Alizarin Red S staining, even in the absence of dexamethasone, and this was associated with significant increases in the activity levels of alkaline phosphatase (ALP), a hydrolase enzyme centrally involved in driving mineralization (Golub and Boesze-Battaglia 2007). These findings are in agreement with previous observations from studies examining the influence of human BMP-2 overexpression on the osteogenic differentiation of equine BMSCs, where increases in ALP activity and mineralization were detected in cells transduced with adenovirus encoding BMP-2 (Carpenter et al. 2010, Murray et al. 2010). However, more recently it has been suggested that osteogenic induction of equine BMSCs is primarily dependent on dexamethasone, rather than BMP-2 (Glynn et al. 2013). It is possible that these contradictory findings may be explained by differences in the concentrations of BMP-2 used.

In addition to increases in mineralization and ALP activity, we also observed significant decreases in secreted osteocalcin levels in response to rhBMP-2. Osteocalcin is well recognized as an inhibitor of hydroxyapatite formation in vitro (Hauschka and Wians 1989, Hunter et al. 1996), and its ablation in vivo results in increased bone formation (Ducy et al. 1996). Therefore, the ability of rhBMP-2 to enhance equine osteoblast mineralization may be directly linked to its inhibitory effects on osteocalcin production. The effects of rhBMP-2 on equine osteoblast differentiation were also evaluated by RT-qPCR analysis of various osteogenic markers including ALP, COL1A2, and RUNX2. Expression levels ALP, which encodes alkaline phosphates, were significantly increased in equine osteoblast cultures in response to osteogenic medium at day 5. In accordance with its stimulatory effects 
on ALP protein activity, treatment with $100 \mathrm{mg} / \mathrm{ml}$ rhBMP-2 induced a significant increase in ALP expression as compared to all other treatment groups. Expression levels of the gene encoding collage type I alpha 2 chain, COL1A2, were significantly reduced in equine osteoblasts in a time dependent manner in response to osteogenic medium, thereby suggesting that reductions in COL1A2 expression favoured osteoblast maturation. This concept is supported by the fact that treatment with $100 \mathrm{mg} / \mathrm{ml}$ rhBMP-2 could further significantly reduce COL1A2 expression in equine osteoblasts at day 5. RUNX2 encodes the Runt-related transcription factor 2 , whose primary role is to direct BMSCs towards the osteoblast lineage, and is subsequently downregulated during osteoblast maturation (Komori 2010). Although we observed significant increases in RUNX2 expression levels in equine osteoblasts incubated in osteogenic medium as compared to controls at both day 2 and 5, treatment with rhBMP-2 failed to induce any significant changes in RUNX2 expression at either time point.

\section{Conclusions}

Treatment of equine SCLs with a combination of debridement and filling with rhBMP-2 in collagen sponges is well tolerated and may be favourable with regards to new subchondral bone formation and improved lameness. Further studies with a larger number of patients and appropriate control groups, may therefore be warranted in order to substantiate these findings and to determine whether rhBMP-2 could be used to treat SCLs at other anatomical locations. The ability of rhBMP-2 to stimulate equine osteoblast differentiation in vitro further highlights its potential use as an osteoinductive agent in horses, and sets the stage for more in-depth investigations into the mechanisms regulating BMP-2-induced equine osteoblast differentiation and mineralization.

\section{Manufacturer's addresses}

a InductOs: Medtronic AG, Münchenbüchsee, Switzerland

b FCR Profect CS, Fujifilm Global, Switzerland

c Somatom Sensation Open, Siemens Medical Solutions, Switzerland

d Equipalazone, MSD Animal Health $\mathrm{GmbH}$, Switzerland

e Thermo Fisher Scientific AG, Reinach, Switzerland

f Sigma-Aldrich Chemie GmbH, Buchs SG, Switzerland

g TECOmedical AG, Sissach, Switzerland

h SPSS Inc., Chicago, IL

\section{Acknowledgements}

The authors would like to thank Aymone Lenisa for her assistance with the in vitro studies, and Wolf-Dieter Wagner and Pascal Bucher for their help with the clinical referrals.

\section{Conflict of interest statement}

None of the authors of this paper has a financial or personal relationship with other people or organisations that could inappropriately influence or bias the content of the paper.

\section{Statement of informed consent}

The owners of the horses presented in the study were informed about the study and gave their consent for the therapy of the SCLs with rhBMP-2.

\section{Funding}

This study was supported in part by the University of Zurich research fund.

\section{References}

Carpenter R. S., Goodrich L. R., Frisbie D. D., Kisiday J. D., Carbone B., Mcllwraith C. W., Centeno C. J., Hidaka C. (2010) Osteoblastic differentiation of human and equine adult bone marrow-derived mesenchymal stem cells when BMP-2 or BMP-7 homodimer genetic modification is compared to BMP-2/7 heterodimer genetic modification in the presence and absence of dexamethasone. J. Orthop. Res. 28, 1330-1337; DOI 10.1002/jor.21126

Cheng H., Jiang W., Phillips F. M., Haydon R. C., Peng Y., Zhou L., Luu H. H., An N., Breyer B., Vanichakarn P., Szatkowski J. P., Park J. Y., He T. C. (2003) Osteogenic activity of the fourteen types of human bone morphogenetic proteins (BMPs). J. Bone Joint Surg. Am. 85-A, 1544-1552

Devescovi V., Leonardi E., Ciapetti G., Cenni E. (2008) Growth factors in bone repair. Chir. Organi Mov. 92, 161-168; DOI 10.1007/s12306-008-0064-1

Ducy P., Desbois C., Boyce B., Pinero G., Story B., Dunstan C., Smith E., Bonadio J., Goldstein S., Gundberg C., Bradley A., Karsenty $G$. (1996) Increased bone formation in osteocalcin-deficient mice. Nature 382, 448-452; DOI 10.1038/382448a0

Fürst A., Kaegi B., von Rechenberg B., Aver J. A. (1997) Die Behandlung von 5 Pferden mit subchondralen zystoiden Defekten im Fesselbein. Pferdeheilkunde 13, 147-161; DOI 10.21836/PEM199 70206

Fürst A., Derungs S., von Rechenberg B., Auer J. A., Schense J., Watson J. (2007) Use of a parathyroid hormone (PTH1-34) enriched hydrogel for the treatment of subchondral cystic lesion in the proximal interphalangeal joint of a warmblood filly: A case report. J. Am. Vet. Med. Assoc. 54, 107-112; DOI 10.1111/j.1439-0442. 2007.00890.x

Glynn E. R., Londono A. S., Zinn S. A., Hoagland T. A., Govoni K. E. (2013) Culture conditions for equine bone marrow mesenchymal stem cells and expression of key transcription factors during their differentiation into osteoblasts. J. Anim. Sci. Biotechnol. 4, 40; DOI 10.1186/2049-1891-4-40

Golub E. E., Boesze-Battaglia K. (2007) The role of alkaline phosphatase in mineralization. Curr. Opin. Orthop. 18, 444-448

Govender S., Csimma C., Genant H. K., Valentin-Opran A., Amit Y., Arbel R., Aro H., Atar D., Bishay M., Borner M. G., Chiron P., Choong P., Cinats J., Courtenay B., Feibel R., Geulette B., Gravel C., Haas N., Raschke M., Hammacher E., van der Velde D., Hardy P., Holt M., Josten C., Ketterl R.L., Lindeque B., Lob G., Mathevon H., Mccoy G., Marsh D., Miller R., Munting E., Oevre S., Nordsletten L., Patel A., Pohl A., Rennie W., Reynders P., Rommens P. M., Rondia J., Rossouw W. C., Daneel P.J., Ruff S., Ruter A., Santavirta S., Schildhaver T. A., Gekle C., Schnettler R., Segal D., Seiler H., Snowdowne R. B., Stapert J., Taglang G., Verdonk R., Vogels L., Weckbach A., Wentzensen A., Wisniewski T., Grp B. S. (2002) Recombinant human bone morphogenetic protein-2 for treatment of open tibial fractures - A prospective, controlled, randomized study of four hundred and fifty patients. Bone Joint Surg. - American Vol. 84a, 2123-2134

Hauschka P. V., Wians F. H. (1989) Osteocalcin-Hydroxyapatite Interaction in the Extracellular Organic Matrix of Bone. Anatomical Record 224, 180-188; DOI 10.1002/ar.1092240208 
Howard R. D., Mcilwraith C. W., Trotter G. W. (1995) Arthroscopic Surgery for Subchondral Cystic Lesions of the Medial Femoral Condyle in Horses - 41 Cases (1988-1991). J. Am. Vet. Med. Ass. 206, 842-850

Hunter G. K., Hauschka P. V., Poole A. R., Rosenberg L. C., Goldberg H. A. (1996) Nucleation and inhibition of hydroxyapatite formation by mineralized tissue proteins. Biochemical J. 317, 59-64

Jackson M. A., Ohlerth S., Kummer M., Bettschart R., Watson J., Manera C. L., von Rechenberg B., Aver J., Fürst A. (2012) Use of a parathyroid hormone peptide (PTH1-34)-enriched fibrin hydrogel for the treatment of subchondral cysticlesions in 15 horses. Pferdeheilkunde 28, 1-6; DOI 10.21836/PEM20120601

Jeffcott L. B, Kold S. E. (1982) Clinical and radiological aspects of stifle bone cysts in the horse. Equine Vet. J. 14, 40-46; DOI 10.1111/j.2042-3306.1982.tb02333.x

Keegan K. G., Dent E. V., Wilson D. A., Janicek J., Kramer J., Lacarrubba A., Walsh D. M., Cassells M. W., Esther T. M., Schiltz P., Frees K. E., Wilhite C. L., Clark J. M., Pollitt C. C., Shaw R., Norris T. (2010) Repeatability of subjective evaluation of lameness in horses. Equine Vet. J. 42, 92-97; DOI 10.2746/042516409X47 9568

Kirker-Head C. A. (1995) Recombinant bone morphogenetic proteins: novel substances for enhancing bone healing. Vet. Surg. 24, 408419; DOI 10.1111/j.1532-950X.1995.tb01352.x

Komori T. (2010) Regulation of bone development and extracellular matrix protein genes by RUNX2. Cell Tissue Res. 339, 189-195; DOI 10.1007/s00441-009-0832-8

McCarthy T. L., Centrella M., Canalis E. (1988) Further Biochemical and Molecular Characterization of Primary Rat Parietal Bone CellCultures. J. Bone Miner. Res. 3, 401-408; DOI 10.1002/jbmr.5650030406

Mcllwraith C. W. (2013) Surgical versus conservative management of osteochondrosis. Vet. J. 197, 19-28; DOI 10.1016/j.tvil.2013. 03.037

Mettenleiter E. (2014) Interventionelle Computertomographie eine neve Dimension in der Pferdemedizin - Teil 1: CT-gesteverte Aufbohrung und Füllung subchondraler Knochenzysten beim Pferd. Pferdeheilkunde 30, 567-576; DOI 10.21836/PEM2014 0508

Mont M. A., Ragland P. S., Biggins B., Friedlaender G., Patel T., Cook S., Etienne G., Shimmin A., Kildey R., Rueger D. C., Einhorn T. A. (2004) Use of bone morphogenetic proteins for musculoskeletal applications - An overview. Bone Joint Surg Am. Vol. 86a, 41-55

Murray S. J., Santangelo K. S., Bertone A. L. (2010) Evaluation of early cellular influences of bone morphogenetic proteins 12 and 2 on equine superficial digital flexor tenocytes and bone marrow-derived mesenchymal stem cells in vitro. Am. J. Vet. Res. 71, 103-114; DOI 10.2460/ajvr.71.1.103

Ortved K. F., Nixon A. J., Mohammed H. O., Fortier L. A. (2012) Treatment of subchondral cystic lesions of the medial femoral condyle of mature horses with growth factor enhanced chondrocyte grafts: a retrospective study of 49 cases. Equine Vet. J. 44, 606-613; DOI 10.1111/j.2042-3306.2011.00510.x

Perrier M., Lu Y., Nemke B., Kobayashi H., Peterson A., Markel M. (2008) Acceleration of second and fourth metatarsal fracture healing with recombinant human bone morphogenetic protein-2/calcium phosphate cement in horses. Vet. Surg. 37, 648-655; DOI 10.1111/j.1532-950X.2008.00431.x

Redding W. (2007) Pathologic conditions involving the internal structures of the foot. In: Equine Podiatry, Eds: A. Floyd and R. Mansmann, Saunders, St. Louis. Pp 253-293

Rubin M. R., Bilezikian J. P. (2003) The anabolic effects of parathyroid hormone therapy. Clin. Geriatr. Med. 19, 415-432; DOI 10.1016/S0749-0690(02)00074-5

Sandhu H. S., Kanim L. E. A., Toth J. M., Kabo J. M., Liv D., Delamarter R. B., Dawson E. G. (1997) Experimental spinal fusion with recombinant human bone morphogenetic protein-2 without decortication of osseous elements. Spine 22, 1171-1180; DOI 10.1097/00007632-199706010-00001
Seo J., Tanabe T., Tsuzuki N., Haneda S., Yamada K., Furuoka H., Tabata Y., Sasaki N. (2013) Effects of bilayer gelatin/beta-tricalcium phosphate sponges loaded with mesenchymal stem cells, chondrocytes, bone morphogenetic protein-2, and platelet rich plasma on osteochondral defects of the talus in horses. Res. in Vet. Science 95, 1210-1216; DOI 10.1016/j.rvsc.2013.08.016

Seo J. P., Kambayashi Y., Itho M., Haneda S., Yamada K., Furuoka H., Tabata Y., Sasaki N. (2015) Effects of a synovial flap and gelatin/beta-tricalcium phosphate sponges loaded with mesenchymal stem cells, bone morphogenetic protein-2, and platelet rich plasma on equine osteochondral defects. Res. In Vet. Science 101, 140-143; DOI 10.1016/j.rvsc.2015.06.014

Seo J. P., Tsuzuki N., Haneda S., Yamada K., Furuoka H., Tabata Y., Sasaki N. (2014) Osteoinductivity of gelatin/beta-tricalcium phosphate sponges loaded with different concentrations of mesenchymal stem cells and bone morphogenetic protein- 2 in an equine bone defect model. Vet. Res. Comm. 38, 73-80; DOI 10.1007/s $11259-013-9587-5$

Sherlock C., Mair T. (2011) Osseous cyst-like lesions/subchondral bone cysts of the phalanges. Equine Vet. Educ. 23, 191-204; DOI 10.1111/i.2042-3292.2010.00222.x

Smith D. M., Cooper G. M., Mooney M. P., Marra K. G., Losee J. E. (2008) Bone morphogenetic protein 2 therapy for craniofacial surgery. J. of Craniofacial Surg. 19, 1244-1259; DOI 10.1097/SCS.0b013e3181843312

Smith M. A., Walmsley J. P., Phillips T. J., Pinchbeck G. L., Booth T. M., Greet T. R., Richardson D. W., Ross M. W., Schramme M. C., Singer E. R., Smith R. K., Clegg P. D. (2005) Effect of age at presentation on outcome following arthroscopic debridement of subchondral cystic lesions of the medial femoral condyle: 85 horses (1993-2003). Equine Vet. J. 37, 175-180; DOI 10.2746/0425 164054223741

Story M. R., Bramlage L. R. (2004) Arthroscopic debridement of subchondral bone cysts in the distal phalanx of 11 horses (19942000). Equine Vet. J. 36, 356-360; DOI 10.2746/042516 4044890643

Sun J., Li J. Y., Li C. C., Yu Y. C. (2015) Role of bone morphogenetic protein-2 in osteogenic differentiation of mesenchymal stem cells. Molec. Med. Rep. 12, 4230-4237; DOI 10.3892/mmr.2015. 3954

Theiss F., Mirsaidi A., Mhanna R., Kummerle J., Glanz S., Bahrenberg G., Tiaden A. N., Richards P. J. (2015) Use of biomimetic microtissue spheroids and specific growth factor supplementation to improve tenocyte differentiation and adaptation to a collagenbased scaffold in vitro. Biomaterials 69, 99-109; DOI 10.1016/ j.biomaterials.2015.08.013

Trotter G., Dyson S. (2003) Osseous cyst like lesions in the distal phalanx. In: Diagnosis and Management of Lameness in the horse, Eds: M. Ross and S. Dyson, Saunders, St. Louis. P 317

Tsuzuki N., Seo J., Yamada K., Haneda S., Furuoka H., Tabata Y., Sasaki N. (2013) The effect of a gelatin beta-tricalcium phosphate sponge loaded with mesenchymal stem cells (MSC), bone morphogenic protein-2, and platelet-rich plasma (PRP) on equine articular cartilage defect. Can. Vet. J. 54, 573-580; DOI 10.1016/ j.rvsc.2011.12.002

Tsuzuki G., Otsuka K., Seo J., Yamada K., Haneda S., Furuoka H., Tabata Y., Sasaki N (2012) In vivo osteoinductivity of gelatin B-tricalcium phosphate sponge and bone morphogenetic protein-2 on an equine third metacarpal bone defect. Res. Vet. Sci. 93, 1021 1025

Verschooten F., De Moor A. (1982) Subchondral cystic and related lesions affecting the equine pedal bone and stifle. Equine Vet. J. 14, 47-54; DOI 10.1111/i.2042-3306.1982.tb02334.x

von Rechenberg B., Guenther H., Mcllwraith C. W., Leutenegger C., Frisbie D. D., Akens M. K., Auer J. A. (2000) Fibrous tissue of subchondral cystic lesions in horses produce local mediators and neutral metalloproteinases and cause bone resorption in vitro. Vet. Surg. 29, 420-429; DOI 10.1053/jvet.2000.7538

von Rechenberg B., Mcllwraith C. W., Auer J. A. (1998) Cystic Bone Lesions in Horses and Humans: A Comparative Review. Vet. Comp. Orthop. Traumatol. 11, 8-18 